\title{
APPROXIMATION OF PARTIAL SUMS OF ARBITRARY I.I.D. RANDOM VARIABLES AND THE PRECISION OF THE USUAL EXPONENTIAL UPPER BOUND
}

\author{
By Marjorie G. Hahn ${ }^{1}$ and Michael J. KLass ${ }^{2}$ \\ Tufts University and University of California, Berkeley
}

This paper quantifies the degree to which exponential bounds can be used to approximate tail probabilities of partial sums of arbitrary i.i.d. random variables. The introduction of a single truncation allows the usual exponential upper bound to apply usefully whenever the summands are arbitrary i.i.d. random variables. More specifically, let $\mathrm{n}$ be a fixed natural number and let $Z, Z_{1}, Z_{2}, \ldots, Z_{n}$ be arbitrary i.i.d. random variables. We construct a function $F_{Z, n}(a)$, derived from the probability of occurrence of one or more "large" summands plus an upper bound of exponential type, such that for some constant $\mathrm{C}_{*}>0$ (independent of $\mathrm{Z}, \mathrm{n}$ and a) and all real $a$,

$$
C_{*} F_{Z, n}^{2}(a) \leq P\left(\sum_{j=1}^{n} Z_{j} \geq n a\right) \leq 2 F_{Z, n}(a) .
$$

Furthermore, examples show that the upper and lower bounds are achievable.

1. Introduction. Let $Z, Z_{1}, Z_{2}, \ldots$ be arbitrary i.i.d. random variables. Consider the problem of approximating $P\left(\sum_{j=1}^{n} Z_{j} \geq n a\right)$ for all $Z, n$ and $a$. Historically, there is some question about whether an approximation of this generality can be obtained. Moreover, issues arise as to the level of precision that can be obtained as the level of generality increases. Most previous results in this direction are asymptotic, in which case it makes sense to replace na by $n a_{n}$. The first such classical result is the central limit theorem. It provides the exact asymptotic magnitude of $P\left(\sum_{j=1}^{n} Z_{j} \geq n a_{n}\right)$ for Z-distributions having finite nonzero variance where $n a_{n}=n E Z+c_{n} \sqrt{n}$ with $c_{n}$ bounded. When $c_{n} \rightarrow \infty$, the central limit theorem merely establishes that $P\left(\sum_{j=1}^{n} Z_{j} \geq n a_{n}\right) \rightarrow 0$, but does not identify the order of magnitude of the rate at which it does so.

As an attempt to extend the range over which such asymptotic approximations could be obtained, large deviations theory was developed. Cramér's (1938) original large deviations theorem, for random variables with a finite moment generating function, provides asymptotic bounds for the behavior of

Received February 1995; revised October 1996.

${ }^{1}$ Supported in part by NSF Grant DMS-87-02878, NSA/MSP Grant MDA-90-H-1008 with NSF Agreement DMS-90-01008, and NSF Grant DMS-92-04333.

${ }^{2}$ Supported in part by NSF Grants DMS-89-06522, DMS-90-07469 and DMS-93-10263.

AMS 1991 subject classifications. Primary 62E 17, 60F 10, 60E 15; secondary 62E 20, 60F 05.

Key words and phrases. Esscher transform, approximation of exceedance levels, nonasymptotic approximations, exponential upper bounds, local probability approximations. 
$(1 / n) \log P\left(\sum_{j=1}^{n} Z_{j} \geq n a_{n}\right)$ where $a_{n} \rightarrow a \in(0, \infty)$. Recent improvements [e.g., a consequence of (i) and (ii) above the remark on page 594 of Ney and Nummelin (1987)] culminate in a result that is valid in general, but gives the relatively uninformative statements that the limit is zero if the tail-probability decay is slower than exponential and the limit is $-\infty$ if its decay is faster than exponential. With some restrictions on $a_{n}$, Theorem 4.1 of $J$ ain and Pruitt (1987) obtains the exact asymptotic magnitude of $P\left(\sum_{j=1}^{n} Z_{j} \geq n a_{n}\right)$ for a large class of nonpositive distributions with stochastically compact normed and centered partial sums. However, the exact order of magnitude of $P\left(\sum_{j=1}^{n} Z_{j} \geq n a_{n}\right)$ has not been obtained in general.

The objective of this paper is to introduce a technique for obtaining, for all $Z$ and $n$, nonasymptotic approximations for $P\left(\sum_{j=1}^{n} Z_{j} \geq n a\right)$ which apply throughout the entire range of the distribution and which always specify the correct order of magnitude of $\log P\left(\sum_{j=1}^{n} Z_{j} \geq n a\right)$. Lemma 2.3 of $J$ ain and Pruitt (1987) is the only other result of which we are aware that attempts to do this in substantial generality. Their result applies to nonpositive distributions for which a is not too close to 0 .

The function we use to approximate the tail probability is obtained from a simple truncation and use of exponential bounds, an idea dating back to at least Fuk and Nagaev (1971). Let $U, U_{1}, U_{2}, \ldots$ be i.i.d. uniform $(0,1)$ random variables. Let $F$ denote the cumulative distribution function of $Z$. Define

Then

$$
F^{-1}(u)=\inf \{z: F(z) \geq u\} \text {. }
$$

$$
L(Z)=L\left(F^{-1}(U)\right)
$$

Hence, we may let $Z=F^{-1}(U)$ and $Z_{j}=F^{-1}\left(U_{j}\right)$. Take a such that $P(Z \geq a)$ $>0$. For any real $0<\mathrm{u} \leq 1$ and $\mathrm{t} \geq 0$,

$$
\begin{aligned}
P\left(\sum_{j=1}^{n} Z_{j} \geq n a\right) \leq & P\left(\bigcup_{j=1}^{n}\left\{U_{j} \geq u\right\}\right) \\
& \left.+P\left(\bigcap_{j=1}^{n}\left\{U_{j}<u\right\}\right) P\left(\sum_{j=1}^{n} Z_{j} \geq n a|| \bigcap_{k=1}^{n}\left\{U_{k}<u\right\}\right]\right) \\
\leq & 1-P^{n}(U<u) \\
& +P^{n}(U<u) E\left(\exp \left(t \sum_{j=1}^{n}\left(Z_{j}-a\right)\right) \mid \bigcap_{k=1}^{n}\left\{U_{k}<u\right\}\right) \\
\leq & 1-P^{n}(U<u)+P^{n}(U<u)\left(E\left(e^{t(Z-a)} \mid U<u\right)\right)^{n} .
\end{aligned}
$$

A similar sort of upper bound can also be found in Fuk and Nagaev (1971).

For each $0<\mathrm{u} \leq 1$ there exists a unique $0 \leq \mathrm{t}_{\mathrm{a}, \mathrm{u}} \leq \infty$ such that

$$
E\left(\exp \left(t_{a, u}(Z-a)\right) \mid U<u\right)=\inf _{t \geq 0} E(\exp (t(Z-a)) \mid U<u) .
$$

Now if there is a $0<u_{a} \leq 1$ such that

$$
E\left(\exp \left(t_{a, u_{a}}(Z-a)\right) \mid U<u_{a}\right)=\left(P^{-n}\left(U<u_{a}\right)-1\right)^{1 / n},
$$


then for $u=u_{a}$ and $t=t_{a} u_{a}$ the two terms in the upper bound in (1.1) would be equal and consequently

$$
\begin{aligned}
P\left(\sum_{j=1}^{n} Z_{j} \geq n a\right) & \leq 2\left(1-P^{n}\left(U<u_{a}\right)\right) \\
& =2 P^{n}\left(U<u_{a}\right)\left(E\left(\exp \left(t_{a}, u_{a}(Z-a)\right) \mid U<u_{a}\right)\right)^{n} .
\end{aligned}
$$

This suggests defining

$$
\begin{aligned}
\mathrm{F}_{\mathrm{Z}, \mathrm{n}}(\mathrm{a}) & =1-\mathrm{P}^{\mathrm{n}}\left(\mathrm{U}<\mathrm{u}_{\mathrm{a}}\right) \\
& =\mathrm{P}^{\mathrm{n}}\left(\mathrm{U}<\mathrm{u}_{\mathrm{a}}\right)\left(\mathrm{E}\left(\exp \left(\mathrm{t}_{\mathrm{a}, \mathrm{u}_{\mathrm{a}}}(\mathrm{Z}-\mathrm{a})\right) \mid \mathrm{U}<\mathrm{u}_{\mathrm{a}}\right)\right)^{\mathrm{n}} \\
& =\left(\mathrm{E} \exp \left(\mathrm{t}_{\mathrm{a}, \mathrm{u}_{\mathrm{a}}}(\mathrm{Z}-\mathrm{a})\right) \mathrm{I}\left(\mathrm{U}<\mathrm{u}_{\mathrm{a}}\right)\right)^{\mathrm{n}} .
\end{aligned}
$$

Moreover, since the left-hand side of (1.3) is at most 1 , it follows that

$$
\mathrm{P}^{\mathrm{n}}\left(\mathrm{U}<\mathrm{u}_{\mathrm{a}}\right) \geq \frac{1}{2} \text {. }
$$

MaIN THEOREM. Fix any integer $n \geq 1$. Let $Z, Z_{1}, Z_{2}, \ldots$ be arbitrary i.i.d. random variables. Take a such that $P(Z \geq a)>0$. There exist a unique $u_{a}$ and $t_{a, u_{a}}$ which simultaneously satisfy (1.2) and (1.3). Define $F_{z, n}(a)$ by (1.5). Then there exists a positive constant $C_{*}$, independent of $Z, n$ and $a$, such that

$$
C_{*} F_{Z, n}^{2}(a) \leq P\left(\sum_{j=1}^{n} Z_{j} \geq n a\right) \leq 2 F_{Z, n}(a) .
$$

Moreover, the upper and lower bounds are achievable

REMARK 1. Observe that

$$
\begin{aligned}
F_{Z, n}(a) & =P^{n}\left(U<u_{a}\right)\left(E\left(\exp \left(t_{a, u_{a}}(Z-a)\right) \mid U<u_{a}\right)\right)^{n} \\
& =P^{n}\left(U<u_{a}\right)\left(\inf _{t \geq 0} E\left(\exp (t(Z-a)) \mid U<u_{a}\right)\right)^{n} \quad \text { by }(1.2) \\
& =\left(\inf _{t \geq 0} E\left(\exp (t(Z-a)) I\left(U<u_{a}\right)\right)\right)^{n} \\
& \leq\left(\inf _{t \geq 0} E(\exp (t(Z-a)))\right)^{n} \wedge P^{n}\left(U<u_{a}\right)
\end{aligned}
$$

Therefore, $F_{z, n}(a)$ is never larger than the usual exponential upper bound, although of course $2 F_{Z, n}(a)$ may be larger. Notice also that in the degenerate case $Z \equiv a, F_{Z, n}(a)=1 / 2$ and so the upper bound for $P\left(\sum_{j=1}^{n} Z_{j} \geq n a\right)$ is attained.

Upper bounds of various sorts are obtained in other papers, including Arkhangel'skii (1989), Bennett (1962), Fuk and Nagaev (1971), Hoeffding (1963), and Pinelis and Utev (1989), where some efforts at optimization were made. Separate upper and lower bounds have been obtained with certain restrictions on the distribution of $Z$ and the range of $a$. See, for example, 
Nagaev (1965), Osipov (1971) and Statulevicius (1966). Asymptotically exact tail probability results for partial sums of i.i.d. random variables in the Feller class may be found in J ain and Pruitt (1987). Also of related interest are the strong large deviation theorems of Chaganty and Sethuraman (1993). For general sequences of random variables (not necessarily restricted to partial sums) having finite moment generating functions, they provide asymptotically exact "tail-probability-like" results under conditions imposed on the moment generating functions. Of course, most large deviations theorems for sums of i.i.d. random variables bear a kinship with the results of this paper. That literature is vast. Consequently, we have tried to confine our citations to works which help to place our results in their proper context.

The lower bound in the Main Theorem will be established in Section 2. Section 3 provides examples to show that the upper and lower bounds are achievable.

2. Proof of the lower bound. The first task is to verify the existence of $\mathrm{u}_{\mathrm{a}}$ and $\mathrm{t}_{\mathrm{a}, \mathrm{u}_{\mathrm{a}}}$ satisfying both (1.2) and (1.3).

Lemma 1. For each real $a$, there exist a unique $u_{a} \in(0,1]$ and a unique $\mathrm{t}_{\mathrm{a}, \mathrm{u}_{\mathrm{a}}} \in[0, \infty]$ which simultaneously satisfy (1.2) and (1.3).

Proof. If $P(Z \geq a)=0$, take $u_{a}=1$ and $t_{a}=\infty$. Now suppose $P(Z \geq a)$ $>0$. Think of the right-hand sides of both (1.2) and (1.3) as functions of $u$ (instead of $u_{a}$ ). It is obvious that the right-hand side of (1.2) is nondecreasing in $u$, always at most 1 , and positive for some $u$. Since the right-hand side of (1.3) strictly decreases as $u$ increases, varying from infinity to zero, whenever $P(Z \geq a)>0$ there exists a unique $u=u_{a}$ at which the left-hand side of (1.2) and right-hand side of (1.3) cross. The two curves will actually intersect with $\mathrm{u}=\mathrm{u}_{\mathrm{a}}$ and $\mathrm{t}=\mathrm{t}_{\mathrm{a}, \mathrm{u}}$ if the function of the left-hand side (equivalently the right-hand side) of (1.2) is continuous in $u$. We consider two cases.

Case $1(P(Z \geq a)=P(Z=a)>0)$. In this case it is readily shown that $\mathrm{t}_{\mathrm{a}, \mathrm{u}} \equiv \mathrm{t}_{\mathrm{a}, \mathrm{u}_{\mathrm{a}}} \equiv \infty$ and

$$
\inf _{t \geq 0} E\left(e^{t(Z-a)} \mid U<u\right)=P(Z=a \mid U<u),
$$

which is obviously continuous in $u$, varying between zero and $P(Z=a)$.

Case $2(P(Z>a)>0)$. Put $\phi_{t}(u)=E\left(e^{t(Z-a)} \mid U<u\right)$. So $\phi_{t}(u)$ is jointly continuous in $0<\mathrm{u}<1$ and $0 \leq \mathrm{t}<\infty$ and nondecreasing in $\mathrm{u}$ for each $\mathrm{t}$. Therefore,

$$
\phi_{*}(\mathrm{u}) \equiv \inf _{\mathrm{t} \geq 0} \phi_{\mathrm{t}}(\mathrm{u})\left(=\inf _{\{\mathrm{t} \geq 0: \mathrm{t} \text { is rational }\}} \phi_{\mathrm{t}}(\mathrm{u})\right)
$$

is upper semicontinuous (i.e., $\left\{0<\mathrm{u}<1: \phi_{*}(u)<w\right\}$ is an open set for every real w) and nondecreasing. As a result, $\phi_{*}(\cdot)$ is right continuous. Continuity of $\phi_{*}(\cdot)$ will follow immediately upon establishing its left-continuity. Take 
any $\mathrm{u}_{*}<\mathrm{u}^{\prime}<\mathrm{u}^{\prime \prime}<1$ such that $\mathrm{F}^{-1}\left(\mathrm{u}_{*}\right)>\mathrm{a}$. If $\mathrm{t}^{\prime}=\mathrm{t}_{\mathrm{a}, \mathrm{u}^{\prime}}$ and $\mathrm{t}^{\prime \prime}=\mathrm{t}_{\mathrm{a}, \mathrm{u}^{\prime \prime}}$, $\phi_{*}\left(\mathrm{u}^{\prime \prime}\right)$

$$
\begin{aligned}
& \leq \mathrm{E}\left(\exp \left(\mathrm{t}^{\prime}(\mathrm{Z}-\mathrm{a})\right) \mid \mathrm{U}<\mathrm{u}^{\prime \prime}\right) \\
& \leq \frac{\mathrm{E} \exp \left(\mathrm{t}^{\prime}(\mathrm{Z}-\mathrm{a})\right) \mathrm{I}\left(\mathrm{U}<\mathrm{u}^{\prime}\right)+\exp \left(\mathrm{t}^{\prime}\left(\mathrm{F}^{-1}\left(\mathrm{u}^{\prime \prime}\right)-\mathrm{a}\right)\right) \mathrm{P}\left(\mathrm{u}^{\prime} \leq \mathrm{U}<\mathrm{u}^{\prime \prime}\right)}{\mathrm{P}\left(\mathrm{U}<\mathrm{u}^{\prime \prime}\right)} \\
& =\frac{\mathrm{P}\left(\mathrm{U}<\mathrm{u}^{\prime}\right)}{\mathrm{P}\left(\mathrm{U}<\mathrm{u}^{\prime \prime}\right)} \phi_{\mathrm{t}^{\prime}}\left(\mathrm{u}^{\prime}\right)+\frac{\exp \left(\mathrm{t}^{\prime}\left(\mathrm{F}^{-1}\left(\mathrm{u}^{\prime \prime}\right)-\mathrm{a}\right)\right)}{\mathrm{P}\left(\mathrm{U}<\mathrm{u}^{\prime \prime}\right)} \mathrm{P}\left(\mathrm{u}^{\prime} \leq \mathrm{U}<\mathrm{u}^{\prime \prime}\right) .
\end{aligned}
$$

Since $\phi_{\mathrm{t}^{\prime}}\left(\mathrm{u}^{\prime}\right) \leq 1$ implies $\exp \left(\mathrm{t}^{\prime}\left(\mathrm{F}^{-1}\left(\mathrm{u}_{*}\right)-\mathrm{a}\right)\right) \mathrm{P}\left(\mathrm{u}_{*} \leq \mathrm{U}<\mathrm{u}^{\prime}\right) \leq 1, \mathrm{t}^{\prime}$ is bounded as $\mathrm{u}^{\prime} \nearrow \mathrm{u}^{\prime \prime}$. Noting also that $\phi_{\mathrm{t}^{\prime}}\left(\mathrm{u}^{\prime}\right)=\phi_{*}\left(\mathrm{u}^{\prime}\right)$, it follows that

$$
\phi_{*}\left(u^{\prime \prime}\right) \leq \limsup _{u^{\prime}>u^{\prime \prime}} \phi_{*}\left(u^{\prime}\right),
$$

whencelim $u_{u^{\prime}>u^{\prime \prime}} \phi_{*}\left(u^{\prime}\right)=\phi_{*}\left(u^{\prime \prime}\right)$. Consequently, $\phi_{*}(u)$ is continuous. Hence, the lemma holds.

Our next objective is to establish that a multiple of the square of the upper bound is in fact a lower bound. This is trivial for $n=1$ and 2 .

When $\mathrm{n}=1$,

$$
P(Z \geq a) \geq P\left(U \geq u_{a}\right)=F_{Z, 1}(a) .
$$

Moreover, when $\mathrm{n}=2$,

$$
\begin{aligned}
P\left(Z_{1}+Z_{2} \geq 2 a\right) & \geq P\left(\bigcap_{j=1}^{2}\left\{Z_{j} \geq a\right\}\right) \\
& \geq P^{2}\left(U \geq u_{a}\right) \\
& \geq \frac{1}{4}\left(1-\left(1-P\left(U \geq u_{a}\right)\right)^{2}\right)^{2} \\
& =\frac{1}{4} F_{Z, 2}^{2}(a) .
\end{aligned}
$$

Hence, the main theorem holds for $\mathrm{n}=1,2$ for any $0<\mathrm{C}_{*} \leq \frac{1}{4}$. The verification for $\mathrm{n} \geq 3$ is more complicated.

Note first that if the lower bound in the main theorem holds for $t_{a, u_{a}}>0, a$ simple monotonicity argument also gives it for $t_{a, u_{a}}=0$. Furthermore, when $\mathrm{t}_{\mathrm{a}, \mathrm{u}_{\mathrm{a}}}=\infty$ [which happens iff $\mathrm{P}(\mathrm{Z}>\mathrm{a})=0$ ], we may conclude from the proof of Lemma 1 [inserted into the last line of (1.5)] that $F_{Z, n}(a)=P^{n}(Z=a, U<$ $\left.u_{a}\right)$; whence $t_{a, u_{a}}=\infty$ implies that $F_{Z, u}^{n}(a) \leq F_{Z, n}(a) \leq P\left(\sum_{j=1}^{n} Z_{j} \geq n a\right)$. So it suffices to consider $0<\mathrm{t}_{\mathrm{a}, \mathrm{u}_{\mathrm{a}}}<\infty$.

For convenience, we first introduce some simplified notation and then make a linear transformation in the random variables. Set $t_{a}=t_{a} u_{a}$. Suppose $0<\mathrm{t}_{\mathrm{a}}<\infty$ and let $\theta_{\mathrm{a}}$ satisfy

$$
\mathrm{E}\left(\exp \left(\mathrm{t}_{\mathrm{a}} \mathrm{Z}\right) \mid \mathrm{U}<\mathrm{u}_{\mathrm{a}}\right)=\exp \left(\mathrm{t}_{\mathrm{a}} \theta_{\mathrm{a}}\right) .
$$

Define

$$
b_{a}=F^{-1}\left(u_{a}\right)=\operatorname{esssup~} L\left(Z \mid U<u_{a}\right)
$$


and set

$$
L(Y)=L\left(\frac{Z-\theta_{a}}{b_{a}-a} \mid U<u_{a}\right)
$$

and

$$
\tilde{\mathrm{t}}_{\mathrm{a}}=\mathrm{t}_{\mathrm{a}}\left(\mathrm{b}_{\mathrm{a}}-\mathrm{a}\right) .
$$

Since $t_{a}>0$, property (1.2) ensures that $\exp \left(t_{a}\left(\theta_{a}-a\right)\right)<1$ so that $\theta_{a}<a$. Moreover, since $t_{a}<\infty, P\left(Z>a \mid U<u_{a}\right)>0$; whence $a<b_{a}$ and so $Y$ is nonconstant. Therefore,

$$
\begin{gathered}
\theta_{a}<a<b_{a}, \\
\text { ess sup } Y=\frac{b_{a}-\theta_{a}}{b_{a}-a} \quad(>1), \\
E \exp \left(\tilde{t}_{a} Y\right)=1, \\
E Y \exp \left(\tilde{t}_{a} Y\right)=\frac{a-\theta_{a}}{b_{a}-a} .
\end{gathered}
$$

Equation (2.8) follows from (1.2) using the quantities defined in (2.1)-(2.4), since the $t=t_{a}$ that minimizes the right-hand side of (1.2) with $u=u_{a}$ satisfies $E\left((Z-a) \exp \left(t_{a}(Z-a)\right) \mid U<u_{a}\right)=0$. Let

$$
\tilde{a}=\frac{a-\theta_{a}}{b_{a}-a} \quad(>0)
$$

and note that

$$
P\left(\sum_{j=1}^{n} Z_{j} \geq n a \mid \bigcap_{k=1}^{n}\left\{U_{k}<u_{a}\right\}\right)=P\left(\sum_{j=1}^{n} Y_{j} \geq n a \tilde{a}\right),
$$

where $Y_{1}, Y_{2}, \ldots$ are i.i.d.

Since $Z \geq b_{a}$ when $U \geq u_{a}$,

$$
\begin{aligned}
P\left(\sum_{j=1}^{n} Z_{j} \geq n a\right) \geq & P\left(\bigcap_{k=1}^{n}\left\{U_{k}<u_{a}\right\}\right) P\left(\sum_{j=1}^{n} Z_{j} \geq n a \mid \bigcap_{k=1}^{n}\left\{U_{k}<u_{a}\right\}\right) \\
+ & \sum_{k=1}^{n}\left(\begin{array}{l}
n \\
k
\end{array}\right) P^{k}\left(U \geq u_{a}\right) P^{n-k}\left(U<u_{a}\right) \\
& \times P\left(\sum_{j=1}^{n-k} Z_{j} \geq n a-k b_{a} \mid \bigcap_{i=1}^{n-k}\left\{U_{i}<u_{a}\right\}\right)
\end{aligned}
$$




$$
\begin{aligned}
& \geq P^{n}\left(U<u_{a}\right) P\left(\sum_{j=1}^{n} Z_{j} \geq n a \mid \bigcap_{k=1}^{n}\left\{U_{k}<u_{a}\right\}\right) \\
&+\sum_{k=1}^{n}\left(\begin{array}{l}
n \\
k
\end{array}\right) P^{k}\left(U \geq u_{a}\right) P^{n-k}\left(U<u_{a}\right) \\
& \quad \times P\left(\sum_{j=1}^{n-1} Z_{j} \geq n a-b_{a} \mid \bigcap_{i=1}^{n-1}\left\{U_{i}<u_{a}\right\}\right) \\
&=P^{n}\left(U<u_{a}\right) P\left(\sum_{j=1}^{n} Y_{j} \geq n a \tilde{}\right) \\
&+\left(1-P^{n}\left(U<u_{a}\right)\right) P\left(\sum_{j=1}^{n-1} Y_{j} \geq(n-1) \tilde{a}-1\right) .
\end{aligned}
$$

The objective is to show that this lower bound is at least a constant multiple of the square of the upper bound in (1.4). Toward this end, we introduce the Esscher transformed variate

$$
\begin{aligned}
\mathrm{P}(\tilde{\mathrm{Y}} \leq \mathrm{y}) & =\left(E \exp \left(\tilde{\mathrm{t}}_{\mathrm{a}} \mathrm{Y}\right)\right)^{-1} \mathrm{E}\left[\exp \left(\tilde{\mathrm{t}}_{\mathrm{a}} \mathrm{Y}\right) \mathrm{I}(\mathrm{Y} \leq \mathrm{y})\right] \\
& =\mathrm{E}\left[\exp \left(\tilde{\mathrm{t}}_{\mathrm{a}} \mathrm{Y}\right) \mathrm{I}(\mathrm{Y} \leq \mathrm{y})\right],
\end{aligned}
$$

by (2.7). Let $\tilde{Y}, \tilde{Y}_{1}, \tilde{Y}_{2}, \ldots$ be i.i.d. with $\tilde{S}_{k}=\tilde{Y}_{1}+\cdots+\tilde{Y}_{k}$. Note that

$$
\begin{aligned}
& P\left(\sum_{j=1}^{n} Y_{j} \geq n \tilde{a}\right) \\
& \quad=\left(E \exp \left(-\tilde{t}_{a} \tilde{S}_{n}\right)\right)^{-1} E\left[\exp \left(-\tilde{t}_{a} \tilde{S}_{n}\right) I\left(\tilde{S}_{n} \geq n a \tilde{a}\right)\right] \\
& \quad=\left(E \exp \left(\tilde{t}_{a}(Y-\tilde{a})\right)\right)^{n} E\left[\exp \left(-\tilde{t}_{a}\left(\tilde{S}_{n}-n \tilde{a}\right)\right) I\left(\tilde{S}_{n} \geq n a \tilde{a}\right)\right]
\end{aligned}
$$

since $E \exp \left(-\tilde{\mathrm{t}}_{\mathrm{a}} \tilde{\mathrm{Y}}\right)=\left(\mathrm{E} \exp \left(\tilde{\mathrm{t}}_{\mathrm{a}} \mathrm{Y}\right)\right)^{-1}$. By (2.8) and (2.9),

$$
\mathrm{EY}=\tilde{a} .
$$

In addition, since $\tilde{Y}$ takes the same values as $Y, \tilde{Y}$ is nonconstant and

$$
\operatorname{ess} \sup (\tilde{Y}-E \tilde{Y})=1
$$

by (2.5), (2.6) and (2.8).

Before finding lower bounds for the terms in the last line of (2.11), we prove a simple approximation lemma.

LEMMA 2. Let $Y$ be a random variable and let $\tilde{\mathrm{t}}>0$ satisfy $E e^{\tilde{t} Y}=1$. Put $\mathrm{P}(\tilde{\mathrm{Y}} \leq \mathrm{y})=\mathrm{Ee}^{\tilde{\mathrm{t}} \mathrm{Y}} \mathrm{I}(\mathrm{Y} \leq \mathrm{y})$ and $\tilde{a}=\mathrm{EY}$. Let $\mathrm{y}_{0} \geq 1$ and $0<\varepsilon<1$ satisfy

$$
1-\varepsilon-\exp \left(-\mathrm{y}_{0}\right)>0 \text {. }
$$


Let

$$
\tilde{\varepsilon}=\frac{\varepsilon+\exp \left(-\mathrm{y}_{0}\right)}{1-\varepsilon-\exp \left(-\mathrm{y}_{0}\right)}
$$

and

$$
d=\tilde{a}+\tilde{\varepsilon}\left(\tilde{a}+\frac{y_{0}}{\tilde{t}}\right)
$$

Then

$$
P\left(-\frac{y_{0}}{\tilde{t}} \leq \tilde{Y} \leq d\right) \geq \varepsilon
$$

Proof. First note that $\tilde{a} \geq 0$. To see this observe that

$$
\tilde{\mathrm{ta}}=\mathrm{E} \tilde{\mathrm{t} Y} \mathrm{e}^{\tilde{\mathrm{t}} \mathrm{Y}}=\mathrm{E}\left((\tilde{\mathrm{t} Y}-1) \mathrm{e}^{\tilde{\mathrm{Y}} Y}+1\right) \geq 0
$$

since $(z-1) e^{z}+1 \geq 0$ for all $z$. Hence $d>0$. Now let $c=-\left(y_{0} / \tilde{t}\right)$.

$$
\begin{aligned}
\tilde{a} & =E \tilde{Y} \geq d P(\tilde{Y} \geq d)+C P(c<\tilde{Y}<d)+E Y e^{\tilde{t} Y} I(Y \leq c) \\
& =d(1-P(\tilde{Y}<d))+C P(c<\tilde{Y}<d)+E Y e^{\tilde{t} Y} I(Y \leq c) \\
& =d-(d-c) P(c<\tilde{Y}<d)-E(d-Y) e^{\tilde{\tilde{Y}}} I(Y \leq c) .
\end{aligned}
$$

Hence

$$
\begin{aligned}
& P(c<\tilde{Y}<d) \geq \frac{d-\tilde{a}}{d-c}-E \frac{d-Y}{d-c} \exp (\tilde{t} Y) I(Y \leq c) \\
& \geq \frac{d-\tilde{a}}{d-c}-\sup _{y \leq c} \frac{d-y}{d-c} \exp (\tilde{t} y) \\
&=\frac{d-\tilde{a}}{d-c}-\exp \left(-y_{0}\right) \\
& \text { since } \frac{\partial}{\partial y}(d-y) \exp (\tilde{t} y) \geq 0 \text { for t̃y } \leq-1 \text { and } d \geq 0 \\
&=\frac{\tilde{\varepsilon}}{1+\tilde{\varepsilon}}-\exp \left(-y_{0}\right) \\
&=1-\exp \left(-y_{0}\right)-\frac{1}{1+\tilde{\varepsilon}} \\
&=\varepsilon
\end{aligned}
$$

Now it is possible to construct lower bounds for the terms in the last line of (2.11). Let $\mathrm{y}_{0} \geq 1$ and $0<\varepsilon<1-\exp \left(-\mathrm{y}_{0}\right)$. Set

$$
\tilde{\varepsilon}=\left(\varepsilon+\mathrm{e}^{-\mathrm{y}_{0}}\right) /\left(1-\varepsilon-\mathrm{e}^{-\mathrm{y}_{0}}\right) .
$$


Let $g_{1}=\left(-2 y_{0} / \tilde{t}_{a}\right)$ and $g_{2}=\tilde{a}+\tilde{\varepsilon}\left(\tilde{a}+\left(2 y_{0} / \tilde{t}_{a}\right)\right)$. Then by (2.11) and the definition of $F_{Z, n}(a)$ given in (1.5),

$$
\begin{aligned}
& \exp \left(2 y_{0}\right)\left(F_{Z, n}(a)\right)^{-2} P\left(\sum_{j=1}^{n} Z_{j} \geq n a\right) \\
& \geq \exp \left(2 y_{0}\right)\left(F_{Z, n}(a)\right)^{-2} P^{n}\left(U<u_{a}\right) P\left(\sum_{j=1}^{n} Y_{j} \geq n a \tilde{}\right) \\
& \quad+\exp \left(2 y_{0}\right)\left(F_{Z, n}(a)\right)^{-2}\left(1-P^{n}\left(U<u_{a}\right)\right) P\left(\sum_{j=1}^{n-1} Y_{j} \geq(n-1) a ̃-1\right)
\end{aligned}
$$

Bounding the first of these two terms from below, we obtain

$$
\begin{aligned}
& \exp \left(2 y_{0}\right)\left(F_{Z, n}(a)\right)^{-2} P^{n}\left(U<u_{a}\right) P\left(\sum_{j=1}^{n} Y_{j} \geq n a \tilde{}\right) \\
& \geq \exp \left(2 y_{0}\right)\left(F_{Z, n}(a)\right)^{-2} P^{2 n}\left(U<u_{a}\right) P\left(\sum_{j=1}^{n} Y_{j} \geq n a ̃\right) \\
& =\exp \left(2 \mathrm{y}_{0}\right) \exp \left(2 \mathrm{t}_{\mathrm{a}} \mathrm{n}\left(\mathrm{a}-\theta_{\mathrm{a}}\right)\right) \mathrm{P}\left(\sum_{\mathrm{j}=1}^{\mathrm{n}} \mathrm{Y}_{\mathrm{j}} \geq \mathrm{na}\right) \quad \text { by (1.5) and (2.1) } \\
& \geq \exp \left(2 \mathrm{y}_{0}\right) \exp \left(\tilde{\mathrm{t}}_{\mathrm{a}} n \tilde{a}\right) E \exp \left(-\tilde{\mathrm{t}}_{\mathrm{a}} \sum_{\mathrm{j}=1}^{\mathrm{n}}\left(\tilde{Y}_{\mathrm{j}}-\tilde{a}\right)\right) \perp\left(\sum_{j=1}^{n} \tilde{Y}_{j} \geq n \tilde{a}\right) \\
& \text { using (2.13) } \\
& \geq \exp \left(\tilde{\mathrm{t}}_{\mathrm{a}}\left(\mathrm{na}+2 \mathrm{y}_{0} \overline{\mathrm{t}}_{\mathrm{a}}^{-1}\right)\right) E \exp \left(-\tilde{\mathrm{t}}_{\mathrm{a}} \sum_{\mathrm{j}=1}^{\mathrm{n}}\left(\tilde{\mathrm{Y}}_{\mathrm{j}}-\tilde{\mathrm{a}}\right)\right) \\
& \times I\left(0 \leq \sum_{j=1}^{n}\left(\tilde{Y}_{j}-\tilde{a}\right) \leq n a \tilde{a}+\frac{2 y_{0}}{\tilde{t}_{a}}\right) \\
& \geq P\left(0 \leq \sum_{j=1}^{n}\left(\tilde{Y}_{j}-\tilde{a}\right) \leq n a \tilde{a}+\frac{2 y_{0}}{\tilde{t}_{a}}\right) \\
& \geq P\left(g_{1} \leq \tilde{Y}_{n} \leq g_{2}\right) \\
& \times P\left(\tilde{a}-g_{1} \leq \sum_{j=1}^{n-1}\left(\tilde{Y}_{j}-E \tilde{Y}\right) \leq(n+1) E \tilde{Y}-g_{2}+\frac{2}{\tilde{t}_{a}} y_{0}\right) \\
& \geq \varepsilon P\left(\tilde{a}+\frac{2 y_{0}}{\tilde{t}_{a}} \leq \sum_{j=1}^{n-1}\left(\tilde{Y}_{j}-E \tilde{Y}\right) \leq(n-\tilde{\varepsilon}) E \tilde{Y}+\frac{2-2 \tilde{\varepsilon}}{\tilde{t}_{a}} y_{0}\right)
\end{aligned}
$$

by Lemma 2 . 
Bounding the second of these two terms from below, we obtain

$$
\begin{aligned}
& \exp \left(2 y_{0}\right) F_{Z, n}^{-2}(a)\left(1-P^{n}\left(U<u_{a}\right)\right) P\left(\sum_{j=1}^{n-1} Y_{j} \geq(n-1) \tilde{a}-1\right) \\
&=\exp \left(2 y_{0}\right) F_{Z, n}^{-1}(a) P\left(\sum_{j=1}^{n-1} Y_{j} \geq(n-1) \tilde{a}-1\right) \text { by }(1.5) \\
&=\exp \left(2 y_{0}+t_{a} n\left(a-\theta_{a}\right)\right) P^{-n}\left(U<u_{a}\right) P\left(\sum_{j=1}^{n-1} Y_{j} \geq(n-1) a ̃-1\right) \\
&= P^{-n}\left(U<u_{a}\right) \exp \left(2 y_{0}+\tilde{t}_{a} \tilde{a}\right) \\
& \times E \exp \left(-\tilde{t}_{a} \sum_{j=1}^{n-1}\left(\tilde{Y}_{j}-E \tilde{Y}\right)\right) I\left(\sum_{j=1}^{n-1}\left(\tilde{Y}_{j}-E \tilde{Y}\right) \geq-1\right) \text { and (2.1) } \\
& \geq P\left(-1 \leq \sum_{j=1}^{n-1}\left(\tilde{Y}_{j}-E \tilde{Y}\right) \leq \tilde{a}+\frac{2 y_{0}}{\tilde{t}_{a}}\right) \\
& \geq \varepsilon P\left(-1 \leq \sum_{j=1}^{n-1}\left(\tilde{Y}_{j}-E \tilde{Y}\right) \leq \tilde{a}+\frac{2 y_{0}}{\tilde{t}_{a}}\right) \quad \text { since } \varepsilon<1 .
\end{aligned}
$$

Therefore, summing the lower bounds obtained in (2.20) and (2.21),

$$
\begin{aligned}
P\left(\sum_{j=1}^{n} Z_{j} \geq n a\right) & \exp \left(2 y_{0}\right) F_{Z, n}^{-2}(a) \\
\geq & \varepsilon P\left(\tilde{a}+\frac{2 y_{0}}{\tilde{t}_{a}} \leq \sum_{j=1}^{n-1}\left(\tilde{Y}_{j}-E \tilde{Y}\right) \leq(n-\tilde{\varepsilon}) E \tilde{Y}+\frac{2-2 \tilde{\varepsilon}}{\tilde{t}_{a}} y_{0}\right) \\
& +\varepsilon P\left(-1 \leq \sum_{j=1}^{n-1}\left(\tilde{Y}_{j}-E \tilde{Y}\right) \leq \tilde{a}+\frac{2 y_{0}}{\tilde{t}_{a}}\right) \\
\geq & \varepsilon P\left(-1 \leq \sum_{j=1}^{n-1}\left(\tilde{Y}_{j}-E \tilde{Y}\right) \leq(n-\tilde{\varepsilon}) E \tilde{Y}+2 \frac{1-\tilde{\varepsilon}}{\tilde{t}_{a}} y_{0}\right) .
\end{aligned}
$$

We may therefore conclude that a constant times the square of the upper bound gives a lower bound provided the probability content of the preceding interval, $\left[-1,(n-\tilde{\varepsilon}) \tilde{a}+\left(2(1-\tilde{\varepsilon}) / \tilde{t}_{a}\right) y_{Q}\right]$, is bounded away from zero relative to the random variable $\sum_{j=1}^{n-1}\left(\tilde{Y}_{j}-E Y\right)$.

Proposition 3. Take any $\mathrm{y}_{0} \geq 2.2$ and $0<\varepsilon<1$ so that $\tilde{\varepsilon}$ from (2.17) satisfies $0<\tilde{\varepsilon} \leq \frac{1}{4}$. Then there exists a constant $\mathrm{C} \equiv \mathrm{C}\left(\tilde{\varepsilon}, \mathrm{y}_{0}\right)>0$ (indepen- 
dent of $\tilde{Y}, \mathrm{n}$ and a) such that for $\mathrm{n} \geq 3$,

$$
P\left(\sum_{j=1}^{n-1}\left(\tilde{Y}_{j}-E \tilde{Y}\right) \in\left[-1,(n-\tilde{\varepsilon}) \tilde{a}+\frac{2(1-\tilde{\varepsilon})}{\tilde{t}_{a}} y_{0}\right]\right)>C .
$$

Proof. To simplify the verification by avoiding randomization at a possible atom on the boundary, it will be assumed that $\tilde{Y}$ is continuous. The proposition remains valid if $\tilde{Y}$ is not continuous and can be derived by representing $\hat{Y}$ as an inverse distribution function of a uniform $(0,1)$ variate. The method by which this can be done is embedded in the proof of Lemma A.1 of the Appendix.

Step 1. Let $\tilde{q}=\sup \{q \geq 0:(n-1) E(\tilde{Y}-\tilde{a} \mid \tilde{Y}-\tilde{a} \geq-q) \geq q\}$. Since $\tilde{Y}$ is assumed to have a continuous distribution,

$$
\tilde{q}=(n-1)(E(\tilde{Y} \mid \tilde{Y} \geq \tilde{a}-\tilde{q})-\tilde{a}) \text {. }
$$

Since $\tilde{Y}$ is non-constant, $\tilde{q}>0$. Let $\tilde{W}, \tilde{W}_{1}, \tilde{W}_{2}, \ldots$ be i.i.d. with

$$
L(\tilde{W})=L(\tilde{Y} \mid \tilde{Y} \geq \tilde{a}-\tilde{q})
$$

and notice that

and

$$
\begin{gathered}
\tilde{a}-\tilde{\mathrm{q}} \leq \tilde{W} \leq \tilde{\mathrm{a}}+1 \quad(\text { since } \tilde{Y} \leq \tilde{\mathrm{a}}+1), \\
(\mathrm{n}-1) E(\tilde{W}-\tilde{Y})=\tilde{\mathrm{q}}, \\
-\frac{n}{n-1} \tilde{\mathrm{q}} \leq \tilde{W}-E \tilde{W} \leq 1-\frac{\tilde{q}}{\mathrm{n}-1}
\end{gathered}
$$

Only (2.29) requires verification: Let $\tilde{p}=P(\tilde{Y} \leq \tilde{a}-\tilde{q})$. Then

$$
\begin{aligned}
\tilde{q} & =(n-1) E \frac{(\tilde{Y}-\tilde{a}) I(\tilde{Y}>\tilde{a}-\tilde{q})}{1-\tilde{p}} \\
& =(n-1) \frac{E(\tilde{a}-\tilde{Y}) I(\tilde{Y} \leq \tilde{a}-\tilde{q})}{1-\tilde{p}} \text { since } E(\tilde{Y}-\tilde{a})=0 \\
& \geq \frac{(n-1) \tilde{q} \tilde{p}}{1-\tilde{p}}
\end{aligned}
$$

Since $\tilde{q}>0$ we have $(n-1) \tilde{p} \leq 1-\tilde{p}$, from which (2.29) follows.

Step 2. Take any $\mathrm{y}_{0} \geq 2.2$ and $0<\varepsilon<1$ so that $\tilde{\varepsilon}$ from (2.17) satisfies $0<\tilde{\varepsilon} \leq \frac{1}{4}$. Then for $\mathrm{n} \geq 3$,

$$
\operatorname{ess} \sup (E \tilde{W}-\tilde{W}) \leq-\tilde{q}+(n-\tilde{\varepsilon}) \tilde{a}+\frac{2(1-\tilde{\varepsilon}) y_{0}}{\tilde{t}_{a}} \text {. }
$$

Proof. By (2.28) it suffices to show that

$$
\frac{2 n-1}{n-1} \tilde{q} \leq(n-\tilde{\varepsilon}) \tilde{a}+\frac{2(1-\tilde{\varepsilon}) y_{0}}{\tilde{t}_{a}} .
$$


If (2.31) fails, then

$$
\begin{aligned}
\tilde{\mathrm{t}}_{\mathrm{a}}(\tilde{\mathrm{q}}-\tilde{\mathrm{a}}) & >\left(\frac{\mathrm{n}^{2}-(3+\tilde{\varepsilon}) \mathrm{n}+1+\tilde{\varepsilon}}{\mathrm{n}(2 \mathrm{n}-1)}\right) \tilde{\mathrm{t}}_{\mathrm{a}} \mathrm{na}+\frac{2(1-\tilde{\varepsilon}) \mathrm{y}_{0}(\mathrm{n}-1)}{2 \mathrm{n}-1} \\
& \geq\left\{\begin{array}{l}
0+\frac{3}{5} \mathrm{y}_{0}, \quad \text { for } \mathrm{n}=3,0<\tilde{\varepsilon} \leq \frac{1}{4}, \\
\frac{1}{7} \tilde{\mathrm{t}}_{\mathrm{a}} \mathrm{na}+\frac{9}{14} \mathrm{y}_{0},
\end{array}\right.
\end{aligned}
$$

We will now obtain an incompatible upper bound of $\tilde{t}_{a}(\tilde{q}-\tilde{a})$. Again, let $\tilde{\mathrm{p}}=\mathrm{P}(\tilde{\mathrm{Y}} \leq \tilde{\mathrm{a}}-\tilde{\mathrm{q}})$. Since $\mathrm{E}(\tilde{\mathrm{Y}}-\tilde{\mathrm{a}})=0$, (2.24) entails

$$
\begin{aligned}
\tilde{q}-\tilde{a} & =\frac{n-1}{1-\tilde{p}} E(\tilde{a}-\tilde{Y}) I(\tilde{Y} \leq \tilde{a}-\tilde{q})-\tilde{a} \\
& \leq \frac{n \tilde{p}-1}{1-\tilde{p}} \tilde{a}+n E\left(-Y e^{\tilde{t}_{a} Y}\right) I(Y \leq \tilde{a}-\tilde{q}) \quad \text { by }(2.29) \\
& \leq n E\left(-Y e^{\tilde{t}_{\mathrm{a}} Y}\right) I(Y \leq \tilde{a}-\tilde{q}) \\
& \text { using (2.29) again and the fact that } \tilde{a}>0 .
\end{aligned}
$$

From here we consider two separate upper bounds. Suppose first that $\mathrm{n}=3$ and $\mathrm{y}_{0}=2.2$. Then

$$
\begin{aligned}
E\left[\left(-Y \mathrm{e}^{\tilde{\mathrm{t}}_{\mathrm{a}} \mathrm{Y}}\right) \mathrm{I}(\mathrm{Y} \leq \tilde{\mathrm{a}}-\tilde{\mathrm{q}})\right] & \leq \sup _{\mathrm{y} \leq \tilde{\mathrm{a}}-\tilde{\mathrm{q}}}-\mathrm{y} \exp \left(\tilde{\mathrm{t}}_{\mathrm{a}} \mathrm{y}\right) \\
& =(\tilde{\mathrm{q}}-\tilde{\mathrm{a}}) \exp \left(-\tilde{\mathrm{t}}_{\mathrm{a}}(\tilde{\mathrm{q}}-\tilde{\mathrm{a}})\right)
\end{aligned}
$$

since $\tilde{t}_{a}(\tilde{q}-\tilde{a})>1$ by (2.32). Consequently, cancelling $\tilde{q}-\tilde{a}$ and using $n=3$,

$$
\exp \left(\tilde{\mathrm{t}}_{\mathrm{a}}(\tilde{\mathrm{q}}-\tilde{\mathrm{a}})\right) \leq 3 \text {. }
$$

This contradicts (2.32) for $\mathrm{y}_{0} \geq \frac{5}{3} \ln 3 \geq=1.831$ (to three significant digits) and we are using $\mathrm{y}_{0}=2.2$.

Next suppose $n \geq 4,0<\tilde{\varepsilon} \leq \frac{1}{4}$, and $y_{0}=2$.2. Let $f(z)=-z /\left(e^{-z}-1+\right.$ z). Then

$$
\begin{aligned}
& E\left(\left(-Y \exp \left(t_{a} Y\right)\right) I(Y \leq \tilde{a}-\tilde{q})\right) \\
& \leq \frac{1}{\tilde{t}_{a}} E\left(\sup _{z \leq \tilde{t}_{a}(\tilde{a}-\tilde{q})} f(z)\left(\left(\tilde{t}_{a} Y-1\right) \exp \left(\tilde{t}_{a} Y\right)+1\right) I(Y \leq(\tilde{a}-\tilde{q}))\right) \\
& \quad \operatorname{since}(Z-1) \exp (z)+1 \geq 0 \quad \forall z \\
& \leq \frac{1}{\tilde{t}_{a}} f\left(\tilde{t}_{a}(\tilde{a}-\tilde{q})\right) E\left(\left(\tilde{t}_{a} Y-1\right) \exp \left(\tilde{t}_{a} Y\right)+1\right) I(Y \leq(\tilde{a}-\tilde{q})) \\
& \quad \text { since } f(z) \text { increases in } z \\
& \leq \frac{1}{\tilde{t}_{a}} f\left(\tilde{t}_{a}(\tilde{a}-\tilde{q})\right) E\left(\left(\tilde{t}_{a} Y-1\right) \exp \left(\tilde{t}_{a} Y\right)+1\right) \\
& =\tilde{a} f\left(\tilde{t}_{a}(\tilde{a}-\tilde{q})\right) \quad \operatorname{since~} E \exp \left(\tilde{t}_{a} Y\right)=1 \text { and } E Y \exp \left(\tilde{t}_{a} Y\right)=\tilde{a} .
\end{aligned}
$$


Inserting (2.34) into inequality (2.33) and cancelling $\tilde{q}-\tilde{a}>0$, it follows that

$$
\begin{aligned}
\exp \left(\tilde{t}_{a}(\tilde{q}-\tilde{a})\right)-1-\tilde{t}_{a}(\tilde{q}-\tilde{a}) & \leq \tilde{t}_{a} n \tilde{a} \\
& <7 \tilde{t}_{a}(\tilde{q}-\tilde{a})-\frac{9}{2} y_{0} \text { by }(2.32)
\end{aligned}
$$

Let $g(w)=e^{w}-1-8 w$. Since $g$ is convex and $g\left(\tilde{t}_{a}(\tilde{q}-\tilde{a})\right)<-\frac{9}{2} y_{0}$,

$$
\begin{aligned}
-9.9 & \geq-\frac{9}{2} \mathrm{y}_{0}>g\left(\tilde{t}_{a}(\tilde{\mathrm{q}}-\tilde{a})\right) \\
& \geq \inf _{\mathrm{w} \geq 0} \mathrm{~g}(\mathrm{w}) \\
& =\mathrm{g}\left(\mathrm{w}^{*}\right) \quad \text { where } \mathrm{w}^{*}=\ln 8 \\
& =7-8 \ln 8=-9.636 \quad \text { (to three significant digits), }
\end{aligned}
$$

which gives a contradiction for $y_{0} \geq 2.2$. Hence, (2.31) holds.

Step 3.

$$
(n-1) \operatorname{Var} \tilde{W} \leq \frac{\tilde{t}_{a} n a ̃(1+\tilde{a})^{2}}{\tilde{t}_{a}(1+\tilde{a})-1+\exp \left(-\tilde{t}_{a}(1+\tilde{a})\right)}
$$

Proof of (2.36). Let $f(z)=z^{2} /\left(z-1+e^{-z}\right)$, with $f(0)=2$. It is readily shown that $f(\cdot)$ is increasing. As usual, let $\tilde{p}=P(\tilde{Y} \leq \tilde{a}-\tilde{q})(\leq(1 / n))$. Then

$$
\begin{aligned}
& (n-1) \operatorname{Var} \tilde{W} \\
& \quad \leq(n-1) E \tilde{W}^{2}=(n-1) \frac{E \tilde{Y}^{2} I(\tilde{Y}>\tilde{a}-\tilde{q})}{1-\tilde{p}} \\
& \quad \leq \frac{n}{\tilde{t}_{a}^{2}} E \tilde{t}_{a}^{2} Y^{2} \exp \left(\tilde{t}_{a} Y\right) I(Y>\tilde{a}-\tilde{q}) \\
& \quad \leq \frac{n}{\tilde{t}_{a}^{2}} E\left(\sup _{z \leq \tilde{t}_{a}(1+\tilde{a})} f(z)\right)\left(\left(\tilde{t}_{a} Y-1\right) \exp \left(\tilde{t}_{a} Y\right)+1\right) I(Y>\tilde{a}-\tilde{q}) \\
& \quad \leq \frac{n}{\tilde{t}_{a}^{2}} f\left(\tilde{t}_{a}(1+\tilde{a})\right) E\left(\left(\tilde{t}_{a} Y-1\right) \exp \left(\tilde{t}_{a} Y\right)+1\right) \quad \text { since } \tilde{Y} \leq \tilde{a}+1 \\
& \quad=\frac{n a \tilde{a}}{\tilde{t}_{a}} f\left(\tilde{t}_{a}(1+\tilde{a})\right) \quad \operatorname{since} E Y \exp \left(\tilde{t}_{a} Y\right)=\tilde{a} \quad \text { and } \quad E \exp \left(\tilde{t}_{a} Y\right)=1 \\
& \quad=\frac{\tilde{t}_{a}(1+\tilde{a})-1+\exp \left(-\tilde{t}_{a}(1+\tilde{a})\right)}{}
\end{aligned}
$$


Step 4. For $\mathrm{y}_{0} \geq \frac{4}{3}, 0<\tilde{\varepsilon} \leq \frac{1}{4}$, and $\mathrm{n} \geq 3$,

$$
\begin{aligned}
& \frac{\tilde{t}_{a} n a \tilde{}(1+\tilde{a})^{2}}{\left(\tilde{t}_{a}(1+\tilde{a})-1+\exp \left(-\tilde{t}_{a}(1+\tilde{a})\right)\right)\left(1+(n-\tilde{\varepsilon}) \tilde{a}+\left(2(1-\tilde{\varepsilon}) y_{0} / \tilde{t}_{a}\right)\right)^{2}} \\
& \quad<\frac{3}{7}
\end{aligned}
$$

Proof OF (2.37). Let $w=\tilde{t}_{a}(1+\tilde{a})$ and $v=\tilde{t}_{a}(n-1-\tilde{\varepsilon}) \tilde{a}$. Then the left-hand side of (2.37) equals

$$
\frac{n}{n-1-\tilde{\varepsilon}} \frac{w^{2} v}{\left(w-1+e^{-w}\right)\left(w+v+2(1-\tilde{\varepsilon}) y_{0}\right)^{2}} \text {. }
$$

The second factor can be bounded as follows:

$$
\begin{aligned}
& \frac{w^{2} v}{\left(w-1+e^{-w}\right)\left(w+v+2(1-\tilde{\varepsilon}) y_{0}\right)^{2}} \\
& \leq \frac{w^{2} v}{\left(w-1+e^{-w}\right)(w+v+2)^{2}} \\
& \leq \frac{w^{2}}{\left(w-1+e^{-w}\right) 4(w+2)} \quad \text { since } \sup _{v>0} \frac{v}{(v+b)^{2}}=\frac{1}{4 b} \quad \text { for all } b>0 \\
& =\frac{w^{2}}{4\left(w^{2}+h(w)\right)} \quad \text { where } h(w)=(w+2)\left(-1+e^{-w}\right)+2 w .
\end{aligned}
$$

Observe that $\mathrm{h}(0)=\mathrm{h}^{\prime}(0)=0$ and $\mathrm{h}^{\prime \prime}(\mathrm{w})=w^{-\mathrm{w}} \geq 0$ for $\mathrm{w} \geq 0$. Hence, $\mathrm{h}(\mathrm{w})$ $>0$ for all $\mathrm{w}>0$, so $\frac{1}{4} \mathrm{w}^{2} /\left(\mathrm{w}^{2}+\mathrm{h}(\mathrm{w})\right)<\frac{1}{4}$. Thus, for $0<\tilde{\varepsilon} \leq \frac{1}{4}$ and $\mathrm{y}_{0} \geq \frac{4}{3}$,

$$
\begin{aligned}
& \frac{\tilde{\mathrm{t}}_{\mathrm{a}} \mathrm{na}(1+\tilde{\mathrm{a}})^{2}}{\left(\tilde{\mathrm{t}}_{\mathrm{a}}(1+\tilde{a})-1+\exp \left(-\tilde{\mathrm{t}}_{\mathrm{a}}(1+\tilde{a})\right)\right)\left(1+(\mathrm{n}-\tilde{\varepsilon}) \tilde{a}+\left(2(1-\tilde{\varepsilon}) \mathrm{y}_{0} / \tilde{\mathrm{t}}_{\mathrm{a}}\right)\right)^{2}} \\
& \quad \leq\left(\frac{\mathrm{n}}{\mathrm{n}-1-\tilde{\varepsilon}}\right) \frac{\mathrm{w}^{2} \mathrm{v}}{\left(\mathrm{w}-1+\exp ^{-\mathrm{w}}\right)\left(\mathrm{w}+\mathrm{v}+2(1-\tilde{\varepsilon}) \mathrm{y}_{0}\right)^{2}} \\
& \quad \leq\left(\frac{\mathrm{n}}{\mathrm{n}-1-\tilde{\varepsilon}}\right) \frac{1}{4} \\
& \quad \leq \frac{3}{7} \text { for } \mathrm{n} \geq 3 .
\end{aligned}
$$

Now let

$$
\begin{aligned}
J & =\left[-1-\tilde{q},-\tilde{q}+(n-\tilde{\varepsilon}) \tilde{a}+\frac{2(1-\tilde{\varepsilon}) y_{0}}{\tilde{t}_{a}}\right] \\
& \equiv\left[-\gamma_{1}, \gamma_{2}\right]
\end{aligned}
$$


and $|\mathrm{J}|=\gamma_{2}+\gamma_{1}$. By Steps 3 and 4

$$
\frac{\operatorname{Var}\left(\sum_{\mathrm{j}=1}^{\mathrm{n}-1}\left(\tilde{\mathrm{W}}_{\mathrm{j}}-E \tilde{W}\right)\right)}{|\mathrm{J}|^{2}}<\frac{3}{7} \text { for } \mathrm{y}_{0} \geq \frac{4}{3} \text {. }
$$

By (2.30),

$$
\operatorname{ess} \sup (E \tilde{W}-\tilde{W}) \leq \gamma_{2} \text { for } y_{0} \geq 2.2
$$

By (2.28),

$$
\operatorname{ess} \sup (\tilde{W}-E \tilde{W})<\gamma_{1} \text {. }
$$

Summing (2.40) and (2.41),

$$
\mid \text { Range }(\tilde{W}-E \tilde{W})\left|<\gamma_{1}+\gamma_{2}=\right| \text { J } \mid \text { for } y_{0} \geq 2.2 \text {. }
$$

By applying (2.40) and (2.41) to Lemma A.1,

$$
P\left(\sum_{j=1}^{n-1}\left(W_{j}-E W_{j}\right) \leq \gamma_{2}\right) \geq 0.05 i
$$

and

$$
P\left(\sum_{j=1}^{n-1}\left(W_{j}-E W_{j}\right) \geq-\gamma_{1}\right) \geq 0.05 .
$$

Hence J intersects the interval between the lowest and highest $5 \%$ of the distribution of $\sum_{\mathrm{j}=1}^{\mathrm{n}}\left(\mathrm{W}_{\mathrm{j}}-\mathrm{EW}_{\mathrm{j}}\right)$.

Combining these two facts with (2.3) and (2.42), Theorem (1.1) of Hahn and Klass (1995) ensures the existence of a constant $\tilde{C}>0$ independent of $n, \tilde{W}$, $\gamma_{1}$, and $\gamma_{2}$ (but depending on 0.05) such that

$$
\mathrm{P}\left(\sum_{\mathrm{j}=1}^{\mathrm{n}-1}\left(\tilde{\mathrm{W}}_{\mathrm{j}}-\mathrm{E} \tilde{\mathrm{W}}\right) \in \mathrm{J}\right) \geq \tilde{\mathrm{C}} \text {. }
$$

Therefore, for $\mathrm{y}_{0} \geq 2.2$ and $\tilde{\varepsilon}=\frac{1}{4}$,

$$
\begin{aligned}
& P\left(\sum_{j=1}^{n-1}\left(\tilde{Y}_{j}-E \tilde{Y}\right) \in\left[-1,(n-\tilde{\varepsilon}) \tilde{a}+\frac{2(1-\tilde{\varepsilon})}{\tilde{t}_{a}} y_{0}\right]\right) \\
& \quad=P\left(\sum_{j=1}^{n-1}\left(\tilde{Y}_{j}-E \tilde{W}\right) \in J\right) \\
& \quad \geq P\left(\bigcap_{i=1}^{n-1}\left\{\tilde{Y}_{j} \geq \tilde{a}-\tilde{q}\right\}\right) P\left(\sum_{j=1}^{n-1}\left(\tilde{W}_{j}-E \tilde{W}\right) \in J\right) \\
& \quad \geq\left(1-\frac{1}{n}\right)^{n-1} \tilde{C} \quad \text { by }(2.29) \text { and }(2.43) \\
& \geq e^{-1} \tilde{C} \equiv C>0,
\end{aligned}
$$

which completes the proof of the proposition. 
3. Sharpness of the bounds. The following example shows that the powers on the upper and lower bounds in the main theorem are sharp. The constant multiplying the lower bound can be no bigger than $\frac{1}{2}((e+1) / e)^{2}$. As noted in Remark 1 (after the main theorem), the constant of 2 in the upper bound is achievable.

EXAMPLE. For each $n \geq 1$, let $X_{n 1}, X_{n 2}, \ldots, X_{n n}$ be i.i.d. random variables with $P\left(X_{n 1}=1\right)=p_{n}=1-P\left(X_{n 1}=0\right)$, where $0<n p_{n} \rightarrow 0$. Let $U$ be uniform on $(0,1)$. We may assume that $X_{n 1}=I\left(1-p_{n}<U<1\right)$. Take any $0<\varepsilon_{\mathrm{n}} \ll 1$ such that $\left(\mathrm{np}_{\mathrm{n}}\right)^{\varepsilon_{\mathrm{n}}} \rightarrow 1$ and let $\alpha_{\mathrm{n}}=1+(-1)^{\mathrm{k}} \varepsilon_{\mathrm{n}}$. Observe that

$$
P\left(\sum_{j=1}^{n} X_{n j} \geq \alpha_{n}\right) \sim \begin{cases}n p_{n}, & \text { if } k=1 \\ \frac{1}{2}\left(n p_{n}\right)^{2}, & \text { if } k=0\end{cases}
$$

We compare the probabilities in (3.1) with the approximation we have devel oped for them. Without loss of generality, we may assume $\left(1-p_{n}\right)^{n}>\frac{1}{2}$. Then for any $0<\alpha_{n}<n$, the analogues of (1.2) and (1.3) can be simultane ously solved by unique $0<t_{n} \equiv t_{n, \alpha_{n} / n}<\infty$ and $u_{n} \equiv u_{n, \alpha_{n} / n} \in\left(1-p_{n}, 1\right)$. Equivalently, the following two equations hold for each such $t_{n}$ and $u_{n}$ :

$$
n E\left[X_{n 1} \exp \left(t_{n} X_{n 1}\right) I\left(U<u_{n}\right)\right]=\alpha_{n} E\left[\exp \left(t_{n} X_{n 1}\right) I\left(U<u_{n}\right)\right]
$$

and

$$
\left(E\left(\exp \left(t_{n} X_{n 1}\right) I\left(U<u_{n}\right)\right)\right)^{n} \exp \left(-t_{n} \alpha_{n}\right)=1-\left(u_{n}\right)^{n} .
$$

From (3.3), (1.5) and the main theorem, our approximation to (3.1) is $1-\left(u_{n}\right)^{n}$. We claim that whether $k=0$ or 1 in the definition of $\alpha_{n}$,

$$
1-\left(u_{n}\right)^{n} \sim \frac{e}{e+1} n p_{n},
$$

whence

$$
\frac{P\left(\sum_{j=1}^{n} X_{n j} \geq 1-\varepsilon_{n}\right)}{1-\left(u_{n}\right)^{n}} \rightarrow \frac{e+1}{e}
$$

and

$$
\frac{P\left(\sum_{j=1}^{n} X_{n j} \geq 1+\varepsilon_{n}\right)}{\left(1-\left(u_{n}\right)^{n}\right)^{2}} \rightarrow \frac{1}{2}\left(\frac{e+1}{e}\right)^{2} .
$$

To establish (3.4), put $y_{n}=e^{t_{n}}$ and $u_{n}=1-\lambda_{n} p_{n}$. Note that $y_{n}>1$, $0<\lambda_{\mathrm{n}}<1$ and

$$
1-\left(u_{n}\right)^{n}=1-\left(1-\lambda_{n} p_{n}\right)^{n} \sim \lambda_{n} n p_{n} .
$$

Since

$$
P\left(X_{n 1} l\left(U<u_{n}\right)=1\right)=u_{n}-1+p_{n}=\left(1-\lambda_{n}\right) p_{n},
$$

(3.2) becomes

$$
n p_{n}\left(1-\lambda_{n}\right) y_{n}=\alpha_{n}\left(1-p_{n}+p_{n}\left(1-\lambda_{n}\right) y_{n}\right) .
$$


From (3.7), $\left(n-\alpha_{n}\right) p_{n}\left(1-\lambda_{n}\right) y_{n}=\alpha_{n}\left(1-p_{n}\right)$. Consequently,

$$
E X_{n 1} \exp \left(t_{n} X_{n 1}\right) I\left(U<u_{n}\right)=p_{n}\left(1-\lambda_{n}\right) y_{n}=\frac{\alpha_{n}\left(1-p_{n}\right)}{n-\alpha_{n}} \sim \frac{1}{n}
$$

Since $E \exp \left(t_{n} X_{n 1}\right) I\left(U<u_{n}\right)=1-p_{n}+E X_{n 1} \exp \left(t_{n} X_{n 1}\right) I\left(U<u_{n}\right)$, inserting (3.8) into (3.3),

$$
\begin{aligned}
\left(1-\mathrm{p}_{\mathrm{n}}+\frac{\alpha_{\mathrm{n}}}{\mathrm{n}-\alpha_{\mathrm{n}}}\left(1-\mathrm{p}_{\mathrm{n}}\right)\right)^{\mathrm{n}} & \sim\left(\mathrm{y}_{\mathrm{n}}\right)^{\alpha_{\mathrm{n}}}\left(1-\left(\mathrm{u}_{\mathrm{n}}\right)^{\mathrm{n}}\right) \\
& \sim\left(\mathrm{y}_{\mathrm{n}}\right)^{\alpha_{\mathrm{n}}} \lambda_{\mathrm{n}} \mathrm{np} \mathrm{p}_{\mathrm{n}} \sim \lambda_{\mathrm{n}}\left(\mathrm{np}_{\mathrm{n}} \mathrm{y}_{\mathrm{n}}\right)^{\alpha_{\mathrm{n}}} .
\end{aligned}
$$

The left-hand side of (3.9) is asymptotically e Hence

$$
\lambda_{\mathrm{n}}\left(\mathrm{np} \mathrm{p}_{\mathrm{n}} \mathrm{y}_{\mathrm{n}}\right)^{\alpha_{\mathrm{n}}} \rightarrow \mathrm{e}
$$

By (3.8), $\left(1-\lambda_{n}\right)^{\alpha_{n}}\left(n p_{n} y_{n}\right)^{\alpha_{n}} \rightarrow 1$, whence $\left(\left(1-\lambda_{n}\right)^{\alpha_{n}} / \lambda_{n}\right) \rightarrow e^{-1}$. A monotonicity argument shows that $\lambda_{\mathrm{n}}$ must be bounded away from 1 . Consequently, $\left(1-\lambda_{n}\right)^{\alpha_{n}-1} \rightarrow 1$ which implies that $\left(1 / \lambda_{n}\right)-1=\left(1-\lambda_{n}\right) / \lambda_{n} \rightarrow$ $\mathrm{e}^{-1}$. Introducing the asymptotic value of $\lambda_{\mathrm{n}}$ into (3.6) yields

$$
1-\left(u_{n}\right)^{n} \sim \lambda_{n} n p_{n} \sim \frac{1}{\mathrm{e}^{-1}+1} \mathrm{np}_{\mathrm{n}}=\frac{\mathrm{e}}{\mathrm{e}+1} \mathrm{np}_{\mathrm{n}},
$$

which is precisely (3.4), and thereby (3.5') and (3.5') follow.

\section{APPENDIX}

Lemma A.1. Let $X, X_{1}, X_{2}, \ldots$ be i.i.d. mean zero random variables with $X_{i} \leq 1$. Then

$$
P\left(\sum_{j=1}^{m} X_{j} \geq-1\right) \geq 0.05>0 .
$$

Proof. We first prove the result for $m=1$ and $m=2$.

$$
\begin{aligned}
\mathrm{P}\left(\mathrm{X} \geq-\frac{1}{\mathrm{~m}}\right) & =1-\mathrm{P}\left(\mathrm{X}^{-}>\frac{1}{\mathrm{~m}}\right) \\
& \geq 1-\mathrm{mEX} X^{-} \\
& =1-\mathrm{mEX} X^{+} \text {since } \mathrm{EX}=0 \\
& \geq 1-\mathrm{mP}\left(\mathrm{X} \geq-\frac{1}{\mathrm{~m}}\right) \quad \text { since } \mathrm{X} \leq 1 .
\end{aligned}
$$

Hence, $P(X \geq-(1 / m)) \geq(m+1)^{-1}$. Therefore,

$$
\begin{aligned}
P\left(\sum_{j=1}^{m} X_{j} \geq-1\right) & \geq P\left(\bigcap_{j=1}^{m}\left\{X_{j} \geq-\frac{1}{m}\right\}\right) \\
& \geq(m+1)^{-m} \geq(9)^{-1} \text { for } m=1 \text { or } 2 .
\end{aligned}
$$


Henceforth, assume $m \geq 3$ and define

$$
\text { (A.2) } \quad q_{m}=\sup \{q \geq 0: m E(X \mid X \geq-q) \geq q\} \text {. }
$$

It may be assumed that $q_{m}>0$ since if $q_{m}=0$, then $P(X=0)=1$ and the lemma obviously holds. Observe that

$$
m E\left(X \mid X \geq-q_{m}\right) \leq q_{m} \leq m E\left(X \mid X>-q_{m}\right) .
$$

As mentioned before, $L(X)$ can be written as $L\left(F^{-1}(U)\right)$ where $U$ is uniform on $(0,1), F$ denotes the cumulative distribution function of $X$, and $F^{-1}(u)=\inf \{y: F(y) \geq u\}$. Let $u_{m, 1}=P\left(X<-q_{m}\right)$ and $u_{m, 2}=F\left(q_{m}\right)=$ $P\left(X \leq-q_{m}\right)$. From (A.3) it follows that

(A.4) $\quad m E\left(F^{-1}(U) \mid U \geq u_{m, 1}\right) \leq q_{m} \leq m E\left(F^{-1}(U) \mid U \geq u_{m, 2}\right)$.

By continuity, there exists $u_{m, 1} \leq u_{m} \leq u_{m, 2}$ such that

$$
\mathrm{mE}\left(\mathrm{F}^{-1}(\mathrm{U}) \mid \mathrm{U} \geq \mathrm{u}_{\mathrm{m}}\right)=\mathrm{q}_{\mathrm{m}} \text {. }
$$

Let $(U, Y)$ be a random vector such that $U$ is uniform on $(0,1)$ and $L(Y)=L\left(F^{-1}(U) \mid U \geq u_{m}\right)$. Clearly, $-q_{m} \leq Y \leq 1$ so that

$$
-\frac{m+1}{m} q_{m} \leq Y-E Y \leq 1-\frac{q_{m}}{m} \text {. }
$$

From (A.6) it follows that

$$
\begin{aligned}
E|Y-E Y|^{3} & \leq(\operatorname{Var} Y) \max \left\{1-\frac{q_{m}}{m},\left(\frac{m+1}{m}\right) q_{m}\right\} \\
& \leq(\operatorname{Var} Y)\left(1+q_{m}\right) .
\end{aligned}
$$

We claim that

$$
(m+1) P\left(U<u_{m}\right) \leq 1 .
$$

PROOF OF (A.8).

$$
\begin{aligned}
\mathrm{q}_{\mathrm{m}} & =m E\left(F^{-1}(\mathrm{U}) \mid \mathrm{U} \geq \mathrm{u}_{\mathrm{m}}\right) \\
& =\frac{m E F^{-1}(\mathrm{U}) \mathrm{I}\left(\mathrm{U} \geq \mathrm{u}_{\mathrm{m}}\right)}{1-\mathrm{P}\left(\mathrm{U}<\mathrm{u}_{\mathrm{m}}\right)} \\
& =\frac{-m E F^{-1}(\mathrm{U}) \mathrm{I}\left(\mathrm{U}<\mathrm{u}_{\mathrm{m}}\right)}{1-\mathrm{P}\left(\mathrm{U}<\mathrm{u}_{\mathrm{m}}\right)} \\
& \geq \frac{m E \mathrm{q}_{\mathrm{m}} \mathrm{I}\left(\mathrm{U}<\mathrm{u}_{\mathrm{m}}\right)}{1-\mathrm{P}\left(\mathrm{U}<\mathrm{u}_{\mathrm{m}}\right)} \text { since } \mathrm{F}^{-1}(\mathrm{u}) \leq-\mathrm{q}_{\mathrm{m}} \text { for } 0<\mathrm{u}<\mathrm{u}_{\mathrm{m}} \\
& =\frac{\mathrm{mP}\left(\mathrm{U}<\mathrm{u}_{\mathrm{m}}\right) \mathrm{q}_{\mathrm{m}}}{1-\mathrm{P}\left(\mathrm{U}<\mathrm{u}_{\mathrm{m}}\right)} .
\end{aligned}
$$

Cancelling $\mathrm{q}_{\mathrm{m}}>0$ and solving for $\mathrm{P}\left(\mathrm{U}<\mathrm{u}_{\mathrm{m}}\right)$ yields $(\mathrm{m}+1) \mathrm{P}\left(\mathrm{U}<\mathrm{u}_{\mathrm{m}}\right) \leq 1$, thereby establishing (A.8). 
Now introduce i.i.d. random vectors $(U, Y),\left(U_{1}, Y_{1}\right), \ldots,\left(U_{n}, Y_{n}\right)$. Putting $\sigma^{2}=\operatorname{Var}(\mathrm{Y})$ and $r_{\mathrm{m}}=\left(1+\mathrm{q}_{\mathrm{m}} / \sigma \sqrt{\mathrm{m}}\right)$,

$$
\begin{aligned}
& P\left(\sum_{j=1}^{m} X_{j} \geq-1\right) \\
& \quad \geq P\left(\bigcap_{k=1}^{m}\left\{U_{k} \geq u_{m}\right\}\right) P\left(\sum_{j=1}^{m} Y_{j} \geq-1\right) \\
& \quad=P{ }^{m}\left(U \geq u_{m}\right) P\left(\frac{\sum_{j=1}^{m}\left(Y_{j}-E Y_{j}\right)}{\sigma \sqrt{m}} \geq \frac{-\left(1+q_{m}\right)}{\sigma \sqrt{m}}\right) \text { by }(A .5) \\
& \quad \geq\left(1-\frac{1}{m+1}\right)^{m} P\left(\frac{\sum_{j=1}^{m}\left(Y_{j}-E Y_{j}\right)}{\sigma \sqrt{m}} \geq-r_{m}\right) \text { by }(A .8) \\
& \quad \geq e^{-1} P\left(\frac{\sum_{j=1}^{m}\left(Y_{j}-E Y_{j}\right)}{\sigma \sqrt{m}} \geq-r_{m}\right) .
\end{aligned}
$$

By the Berry-Esseen Theorem,

$$
\begin{aligned}
P\left(\frac{\sum_{j=1}^{m}\left(Y_{j}-E Y_{j}\right)}{\sigma \sqrt{m}} \geq-r_{m}\right) & \quad \text { where the Berry-Esseen constant } c_{*} \leq 0.7975 \\
\geq & \int_{-r_{m}}^{\infty} \frac{1}{\sqrt{2 \pi}} \exp \left(-y^{2} / 2\right) d y-c_{*} \frac{m E|Y-E Y|^{3}}{(\sigma \sqrt{m})^{3}} \\
\geq & \frac{1}{2}+\int_{0}^{r_{m}} \frac{1}{\sqrt{2 \pi}} \exp \left(-y^{2} / 2\right) d y \\
& -\frac{0.7975}{\sigma \sqrt{m}} \max \left\{1-\frac{q_{m}}{m},\left(\frac{m+1}{m}\right) q_{m}\right\} \quad \text { by }(A .7) \\
\geq & \frac{1}{2}-(0.7975) r_{m}+\int_{0}^{r_{m}} \frac{1}{\sqrt{2 \pi}} \exp \left(-y^{2} / 2\right) d y \equiv g_{1}\left(r_{m}\right) .
\end{aligned}
$$

[See Van Beeck (1972) for the bound on the Berry-Esseen constant used above.] Note that $g_{1}(r)$ decreases, $g_{1}(0)=\frac{1}{2}$ and $g_{1}(\infty)=-\infty$.

By Marshall's inequality [Marshall (1960)],

$$
P\left(\frac{\sum_{j=1}^{m}\left(Y_{j}-E Y_{j}\right)}{\sigma \sqrt{m}} \geq-r_{m}\right) \geq \frac{\left(r_{m}\right)^{2}}{1+\left(r_{m}\right)^{2}} \equiv g_{2}\left(r_{m}\right) \text {. }
$$

Note that $g_{2}(r)$ strictly increases on $0 \leq r \leq \infty, g_{2}(0)=0$, and $g_{2}(\infty)=1$. Consequently, there exists a unique $0<r^{*}<\infty$ such that $g_{1}\left(r^{*}\right)=g_{2}\left(r^{*}\right)$. In particular, this implies that

$$
\inf _{r>0} \max \left\{g_{1}(r), g_{2}(r)\right\}=\sup _{r>0} \min \left\{g_{1}(r), g_{2}(r)\right\} \text {. }
$$


Hence,

$$
\begin{aligned}
P\left(\sum_{j=1}^{m} Y_{j} \geq-1\right) & \geq e^{-1} \max \left\{g_{1}\left(r_{m}\right), g_{2}\left(r_{m}\right)\right\} \geq e^{-1} \inf _{r>0} \max \left\{g_{1}(r), g_{2}(r)\right\} \\
& =e^{-1} \sup _{r>0} \min \left\{g_{1}(r), g_{2}(r)\right\} \geq e^{-1} \min \left\{g_{1}(0.4), g_{2}(0.4)\right\} \\
& =e^{-1} g_{2}(0.4) \geq 0.05>0 .
\end{aligned}
$$

\section{REFERENCES}

ARKHANGEL'SKII, A. N. (1989). Lower bounds for probabilities of large deviations for sums of independent random variables. Theory Probab. Appl. 34 565-575.

Bennett, G. (1962). Probability inequalities for sums of independent random variables. J . Amer. Statist. Assoc. 57 33-45.

Chaganty, N. R. and Sethuraman, J . (1993). Strong large deviation and local limit theorems. Ann. Probab. 21 1671-1690.

Cramér, H. (1938). On a new limit theorem in the theory of probability. In Colloquium on the Theory of Probability. Hermann, Paris.

FuK, D. KH. and NagaeV, S. V. (1971). Probability inequalities for sums of independent random variables. Theory Probab. Appl. 16 643-660.

HAHN, M. G. and KLASS, M. J . (1995). Uniform local probabilities approximations: improvements on Berry-Esseen. Ann. Probab. 23 446-463.

Hoeffing, W. (1963). Probability inequalities for sums of bounded random variables. J . Amer. Statist. Assoc. 58 13-30.

J AIN, N. C. and PRUITT, W. E. (1987). Lower tail probability estimates for subordinators and nondecreasing random walks. Ann. Probab. 15 75-102.

MARShalL, A. W. (1960). A one-sided analog of Kolmogorov's inequality. Ann. Math. Statist. 31 $483-487$.

Nagaev, S. V. (1965). Some limit theorems for large deviations. Theory Probab. Appl. 10 214-235.

Ney, P. and Nummelin, E. (1987). Markov additive processes II. Large deviations. Ann. Probab. 15 593-609.

Osıpov, A. (1971). On asymptotic expansions for the distributions of sums of independent random variables. Theory Probab. Appl. 16 333-343.

PINELIS, I. S. and UTEV, S. A. (1989). Exact exponential bounds for sums of independent random variables. Theory. Probab. Appl. 34 340-346.

Statulevicius, V. A. (1966). On large deviations. Z. Wahrsch. Verw. Gebiete 6 133-144.

VAN BEECK, P. (1972). An application of Fourier methods to the problem of sharpening the Berry-Esseen inequality. Z. Wahrsch. Verw. Gebiete 23 187-196.

Department of Mathematics

TUFTS UNIVERSITY

MedFoRd, MASSACHUSETTS 02155
Department of Statistics

UNIVERSITY OF CALIFORNIA

BERKELEY, CALIFORNIA 94720

E-MAIL: klass@stat.berkeley.edu 